Pathologe 2018 · 39 (Suppl 2):S306-S310 https://doi.org/10.1007/s00292-018-0542-7 Online publiziert: 5 . November 2018

(c) Der/die Autor(en) 2018

CrossMark

\section{T. Ripperger $\cdot$ B. Schlegelberger}

Institut für Humangenetik, Medizinische Hochschule Hannover, Hannover, Deutschland

\title{
Genetische Prädisposition für Krebserkrankungen im Kindesalter
}

Beim Thema genetische Prädisposition für Krebserkrankungen im Kindesalter ist eine enge Kooperation zwischen $\mathrm{Pa}$ thologen, den behandelnden Kinderonkologen und Humangenetikern erforderlich. Dabei geht es nicht nur um die möglichst effektive Behandlung der Krebserkrankung, sondern auch darum, weitere Krebserkrankungen bei den Betroffenen und ihren Familienmitgliedern zu verhindern bzw. möglichst früh zu diagnostizieren.

Folgende Kriterien legen den Verdacht nahe, dass eine Krebserkrankung im Kindesalter auf der Basis einer genetischen Prädisposition entstanden ist:

- Mehrere Krebserkrankungen in der Familie

- Auffälligkeiten an anderen Organen oder syndromale Merkmale

- Exzessive Toxizität

- Multiple unabhängige Tumoren

- Bilaterale Tumoren

- Spezifische histopathologisch definierte Tumortypen

- Somatische Mutationen in Genen, in denen konstitutionelle Varianten bekannt sind

Diese Kriterien werden inzwischen differenziert in einem Fragebogen erfasst, der in allen zertifizierten Kinderkrebskliniken eingesetzt wird und auf den Übersichtsarbeiten von Jongmanns et al. und Ripperger et al. beruht $[5,9]$. Somit ist zu erwarten, dass in den nächsten Jahren zunehmend mehr Kinder und Jugendliche mit einer genetischen Krebsprädisposition identifiziert werden.

Etwa 5-10\% aller Krebserkrankungen im Kindes- und Jugendalter sind auf eine Keimbahnmutation zurückzuführen
[3]. Hier gibt es deutliche Unterschiede zwischen den Entitäten. Während mehr als $70 \%$ der Kinder mit einem myelodysplastischen Syndrom (MDS) mit Monosomie 7, 50 \% der Kinder mit adrenokortikalen Karzinomen und etwa ein Drittel der Kinder mit einem malignen Rhabdoidtumor eine prädisponierende Keimbahnvariante tragen, wurden bei $6 \%$ der Kinder mit einem Medulloblastom Keimbahnvarianten nachgewiesen $[1,3,19,20]$. Das am häufigsten betroffene Gen bei Kindern mit einem Medulloblastom ist TP53. In diesen Fällen liegt ein Li-Fraumeni-Syndrom vor. Diese Erkrankung ist durch früh auftretende Krebserkrankungen, insbesondere adrenokortikale Karzinome, Plexus-choroideus-Karzinome, Sarkome und Brustkrebs charakterisiert. Auch andere sog. Konsensusgene für $\mathrm{Me}$ dulloblastomprädispositionen, wie zum Beispiel APC, BRCA2, PALB2, PTCH1, und $S U F U$, sind Schlüsselgene für bekannte erbliche Krebserkrankungen, die familiäre adenomatöse Polypose (FAP), den erblichen Brust- und Eierstockkrebs (HBOC) sowie das Gorlin-GoltzSyndrom [19].

Somatische und konstitutionelle Veränderungen finden sich häufig in denselben Genen. Beim Neuroblastom, der akuten lymphatischen Leukämie und dem MDS bzw. der akuten myeloischen Leukämie (AML) lässt sich der Zusammenhang zwischen somatischen und konstitutionellen Veränderungen eindrucksvoll belegen. Die Gene, in denen Keimbahnvarianten identifiziert wurden, sind diejenigen, die auch gehäuft

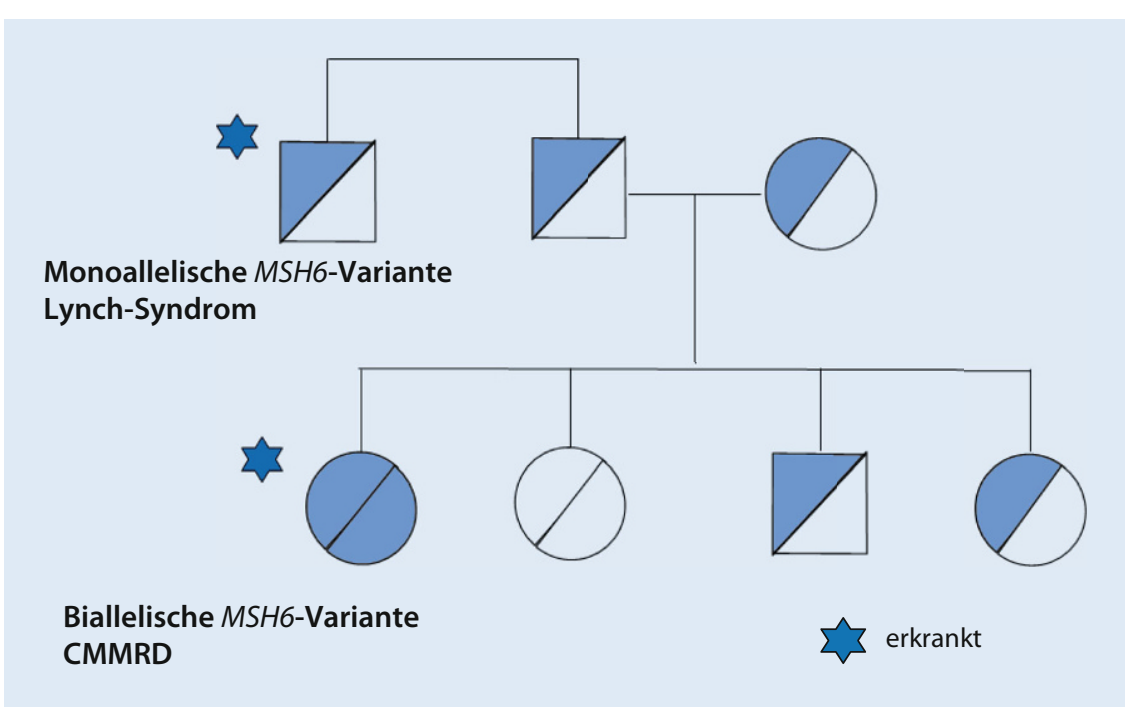

Abb. $1 \Delta$ Die konstitutionelle Mismatch-Repair-Defizienz (CMMRD) ist auf die biallelische Inaktivierung in einem Mismatch-Repair-Gen, wiez. B.MSH6, zurückzuführen. Die Eltern und weitere Verwandte tragen eine monoallelische (heterozygote) pathogene Variante und haben damit ein Lynch-Syndrom 
Tab. 1 Beispiele für somatische und konstitutionelle Varianten in Krebserkrankungen des Kindesalters

\begin{tabular}{|c|c|c|c|c|}
\hline Erkrankung & Gen & $\begin{array}{l}\text { Somatische } \\
\text { Varianten }\end{array}$ & $\begin{array}{l}\text { Konstitutionelle Vari- } \\
\text { anten }\end{array}$ & Referenzen \\
\hline $\begin{array}{l}\text { Neuro- } \\
\text { blastom }\end{array}$ & $A L K$ & Fusionsgene & $\begin{array}{l}\text { Hotspotmutation } \\
\text { c.3824G }>\text { A } \\
\text { p. }(\text { Arg1275GIn) }\end{array}$ & $\begin{array}{l}\text { Mosse et al. } 2008 \text { [6], } \\
\text { Tolbert et al. } 2017 \text { [17] }\end{array}$ \\
\hline \multirow[t]{2}{*}{ ALL } & PAX5 & Deletionen & $\begin{array}{l}\text { Hotspotmutation } \\
\text { c.547G }>\text { A p.(Gly } 183 \text { Ser) }\end{array}$ & Shah et al. 2013 [15] \\
\hline & IKZF1 & Deletionen & $\begin{array}{l}28 \text { unterschiedliche } \\
\text { inaktivierende Varianten }\end{array}$ & $\begin{array}{l}\text { Churchman et al. } 2018 \\
\text { [2], Stanulla et al. } 2018 \\
\text { [16] }\end{array}$ \\
\hline \multirow[t]{3}{*}{ AML/MDS } & RUNX1 & $\begin{array}{l}\text { Missense- } \\
\text { varianten, } \\
\text { Fusionsgene }\end{array}$ & $\begin{array}{l}\text { Missense- und Frame- } \\
\text { shiftvarianten, Exon-/ } \\
\text { Gendeletionen, introni- } \\
\text { sche Varianten }\end{array}$ & $\begin{array}{l}\text { Ripperger et al. } 2009 \\
\text { [11], Ripperger et al. } \\
2011 \text { [12] }\end{array}$ \\
\hline & GATA2 & $\begin{array}{l}\text { Missense- } \\
\text { varianten }\end{array}$ & $\begin{array}{l}\text { Missense- und Frame- } \\
\text { shiftvarianten, Exon-/ } \\
\text { Gendeletionen, introni- } \\
\text { sche Varianten }\end{array}$ & Wlodarski et al. 2016 [20] \\
\hline & $\begin{array}{l}\text { MECOM } \\
\text { (EVI1) }\end{array}$ & $\begin{array}{l}\text { Inversion } 3 \mathrm{q} \text {, } \\
\text { Translokationen }\end{array}$ & Missense & Ripperger et al. 2018 [10] \\
\hline
\end{tabular}

Tab. 2 Drei unabhängige Krebserkrankungen bei einem Kind mit einer pathogenen Variante im TP53-Gen und damit einem Li-Fraumeni-Syndrom. (Nach Schlegelberger et al. [14])

\begin{tabular}{|c|c|c|}
\hline Alter & Maligne Erkrankung & Behandlung \\
\hline 4 Monate & Plexus-choroideus-Karzinom & Operation, Temozolamid \\
\hline 4,2 Jahre & Akute myeloische Leukämie & $\begin{array}{l}\text { Chemotherapie nach dem } \\
\text { AML-BFM-Protokoll; Stamm- } \\
\text { zelltransplantation (Fremd- } \\
\text { spender) }\end{array}$ \\
\hline 5,6 Jahre & $\begin{array}{l}\text { Schlecht differenziertes Nephroblastom } \\
\text { (Wilms-Tumor) }\end{array}$ & Operation \\
\hline 6 Jahre & Vollremission & - \\
\hline
\end{tabular}

Tab. 3 Beginn der Früherkennungsuntersuchungen für Constitutional-Mismatch-Repair-Defizienz(CMMRD)- und Lynch-Syndrom im Vergleich. (Nach Jarvinen et al. [4] und Vasen et al. [18])

\begin{tabular}{|l|l|l|}
\hline Maßnahme & CMMRD & Lynch \\
\hline Klinische Untersuchung & 1 Jahr & 25 Jahre \\
\hline Ultraschall Oberbauch & 1 Jahr & 25 Jahre \\
\hline Magnetresonanztomographie Kopf & 2 Jahre & - \\
\hline Komplette Koloskopie & 8 Jahre & 25 Jahre \\
\hline Ösophagogastroduodenoskopie & 10 Jahre & 35 Jahre \\
\hline Transvaginaler Ultraschall & 20 Jahre & 25 Jahre \\
\hline Endometriumbiopsie & 20 Jahre & 35 Jahre \\
\hline $\begin{array}{l}{ }^{a} \text { Oder 5 Jahre vor dem frühesten Erkrankungsalter in der Familie } \\
\text { CMMRD Constitutional-Mismatch-Repair-Defizienz }\end{array}$ \\
\hline
\end{tabular}

von somatischen Mutationen betroffen sind (•Tab. 1). Keimbahnvarianten können dabei Punktmutationen und kleine Indels in codierenden Regionen eines Gens darstellen bzw. Bereiche betreffen, die für das korrekte Splicen verantwort- lich sind. Es finden sich jedoch auch Deletionen einzelner Exons, größere Deletionen, die das gesamte Gen bzw. mehrere Gene umfassen sowie Alterationen in nichtcodierenden Bereichen. Ein Beispiel dafür ist ein Polymorphis- mus im ersten Intron des LMO1-Gens innerhalb eines „super-enhancers“, das die Bindung von Transkriptionsfaktoren verändert und die Expression des LMO1-Gens beeinflusst. Dies führt zu einer erhöhten Suszeptibilität für Neuroblastome und in den Tumorzellen zu einer Abhängigkeit von diesem Onkogen [7].

Bestimmte histopathologisch definierte Tumorentitäten sind pathognomonisch für spezifische genetische Tumorprädispositionssyndrome. Beim Li-FraumeniSyndrom sind dies das Plexus-choroideus-Karzinom, das adrenokortikale Karzinom, das embryonale Rhabdomyosarkom; beim DICER1-Syndrom das pleuropulmonale Blastom oder der Sertoli-Leydig-Zell-Tumor des Ovars. Daher ist eine differenzierte histopathologische Diagnostik bei diesen seltenen Tumoren des Kindesalters unerlässlich. In vielen Fällen gibt die histopathologische Diagnose den ersten Hinweis, dass ein genetisches Tumorprädispositionssyndrom vorliegen könnte und weist den Weg bei der Suche nach der ursächlichen Keimbahnvariante [9]. Beim Constitutional-Mismatch-RepairDefizienz(CMMRD)-Syndrom lässt der komplette immunhistochemische Ausfall eines Mismatch-Repair-Enzyms (d.h. PMS2, MSH6, MLH1 oder MSH2) auf eine homozygote Inaktivierung des entsprechenden Gens schließen [8].

Die Identifizierung eines genetischen Tumorprädispositionssyndroms hat weitreichende klinische Konsequenzen für den Patienten und seine gesamte Familie.

- Unmittelbare Konsequenzen für die

Therapie:

Beim Li-Fraumeni-Syndrom und anderen DNA-Reparaturdefekten sollte auf die Bestrahlung wenn möglich verzichtet werden, da es ernst zu nehmende Hinweise gibt, dass durch die Strahlentherapie Sarkome induziert werden. Bei der RUNX1-assoziierten familiäre Plättchenerkrankung mit Neigung zu myeloischen Neoplasien (FPDMM) drohen schwerwiegende Komplikationen nach der Stammzelltransplantation, wenn Träger der familiären Variante als Familienspender dienen [13]. Bei Patienten mit CMMRD- und POLE-assoziierten 
Erkrankungen wird der Einsatz von Immun-Checkpoint-Inhibitoren in klinischen Studien geprüft.

- Erhöhtes Risiko für sekundäre Tumoren:

Je jünger Patienten mit Li-Fraumeni-Syndrom erkranken, umso höher ist das Risiko für weitere unabhängige Krebserkrankungen. Als Beispiel wurde ein Kind mit 3 unabhängigen Tumoren präsentiert (- Tab. 2; [14]). Daher sollten Kinder und Erwachsene mit genetischen Krebsprädispositionssyndromen in altersadaptierte, an das spezifische Risiko angepasste Früherkennungsprogramme integriert werden. Das gilt auch für Patienten nach einer erfolgreich behandelten Tumorerkrankung. Für Frauen mit einer pathogenen TP53-Keimbahnvariante ist die Wahrscheinlichkeit, früh an Brustkrebs zu erkranken, sehr hoch. Daher sollten sie in ein intensiviertes Früherkennungsprogramm eingeschleust werden. Auch eine prophylaktische Mastektomie sollte mit den Patientinnen diskutiert werden.

- Prädiktive Diagnostik bei Familienmitgliedern:

Die meisten Keimbahnvarianten für genetische Tumorprädispositionssyndrome werden nach dem autosomal-dominanten Erbgang vererbt. Ausnahmen im Kindesalter sind z. B. die CMMRD und Blutbildungsstörungen wie die FanconiAnämie, die mit einem erhöhten Risiko für MDS/AML und Plattenepithelkarzinome einhergeht. Diese Erkrankungen werden autosomal-rezessiv vererbt. Die Betroffenen tragen eine pathogene homozygote Variante bzw. zwei komplex heterozygot vorliegende pathogene Varianten. Die Eltern sind in der Regel Träger einer entsprechend heterozygoten Variante (- Abb. 1). Bei der CMMRD bedeutet dies, dass die Eltern formal ein LynchSyndrom haben und damit ein deutlich erhöhtes Risiko für Darmkrebs und bei Frauen auch für Endometriumkarzinome besteht. Zudem liegen erhöhte Erkrankungswahrscheinlichkeiten für weitere Lynch-Syndromassoziierte Tumoren vor. Nach den

Pathologe 2018 - 39 (Suppl 2):S306-S310 https://doi.org/10.1007/s00292-018-0542-7

(c) Der/die Autor(en) 2018

\section{T. Ripperger · B. Schlegelberger}

\section{Genetische Prädisposition für Krebserkrankungen im Kindesalter}

\section{Zusammenfassung}

Beim Thema genetische Prädisposition für Krebserkrankungen im Kindesalter ist eine enge Kooperation zwischen Pathologen, behandelnden Kinderonkologen und Humangenetikern erforderlich. Dabei geht es nicht nur um die präzise Diagnose und möglichst effektive Behandlung der Krebserkrankung, sondern auch darum, weitere Krebserkrankungen bei den Betroffenen und ihren Familienmitgliedern zu verhindern bzw. möglichst früh zu diagnostizieren. Anhand von Beispielen wie dem Li-FraumeniSyndrom, der konstitutionellen MismatchRepair-Defizienz (CMMRD), dem Medullo- und Neuroblastom sowie hämatologischen Neoplasien werden die Kriterien für genetische Tumorprädispositionssyndrome, der Zusammenhang zwischen somatischen Varianten und Keimbahnvarianten sowie die unmittelbaren klinischen Konsequenzen erörtert. Bei einigen genetischen Tumorprädispositions- syndromen hat die Diagnose unmittelbare Konsequenzen für die Behandlung, z. B. den Verzicht auf eine Strahlentherapie beim LiFraumeni-Syndrom. Es besteht eine deutlich erhöhte Wahrscheinlichkeit für sekundäre, unabhängig entstandene Tumoren. Durch die prädiktive Diagnostik können die Familienmitglieder identifiziert werden, die die pathogene Variante tragen und aufgrund ihres erhöhten Tumorrisikos das Angebot einer intensivierten Früherkennung oder ggf. prophylaktischer Operationen erhalten sollten. Evidenzbasierte Daten zeigen, dass Programme zur intensivierten Früherkennung zu einer signifikanten Verbesserung der Überlebenswahrscheinlichkeitführen.

\section{Schlüsselwörter}

DNA-Mismatch-Reparatur - Genetische Prädisposition - Genetische Beratung · Krebsfrüherkennung $\cdot$ Li-Fraumeni-Syndrom

\section{Genetic predisposition to childhood cancer}

\section{Abstract}

Tackling the topic of genetic predisposition to childhood cancer requires close co-operation between pathologists, pediatric oncologists, and human geneticists. It is not just about the precise diagnosis and the most effective treatment of the cancer, but also to prevent further cancerous diseases for those affected and also their family members. On the basis of examples such as Li-Fraumeni syndrome, constitutional mismatch repair deficiency (CMMRD), medullo- and neuroblastoma, as well as hematological neoplasias, we will discuss the criteria for tumor predisposition genetic syndromes, the relationship between somatic and germline variants, and the immediate clinical consequences. In some cases, the diagnosis of a genetic tumor predisposition syndrome has immediate consequences for the treatment, e.g. to avoid radiotherapy for Li-Fraumeni syndrome, which would otherwise significantly increase the probability of secondary, independent tumors. Predictive diagnostics can be offered to identify the family members who carry the pathogenic variant. Because of their increased tumor risk, they should be integrated into cancer surveillance programs. Evidence-based data show that this significantly improves overall survival.

Keywords

DNA mismatch repair - Genetic predisposition to disease - Genetic counselling - Early detection of cancer - Li-Fraumeni syndrome
Empfehlungen der Deutschen Gesellschaft für Stoffwechselerkrankungen ist in diesem Fall ein intensiviertes Früherkennungsprogramm und ggf. eine prophylaktische Hysterektomie anzubieten. Evidenzbasierte Daten zeigen, dass die intensivierte Darmkrebsfrüherkennung mit einer signifikanten Verbesserung der Über- lebenswahrscheinlichkeit verbunden ist (• Tab. 3).

Bei genetischen Tumorprädispositionssyndromen kann durch eine prädiktive Diagnostik nach adäquater genetischer Beratung geklärt werden, wer in der Familie ebenfalls die pathogene(n) Variante(n) trägt. Angehörige, die die Genveränderung(en) nicht tragen, können hierdurch 


\section{Infobox Relevante Internet- adressen}

- https://www.krebsgesellschaft.de/

- https://www.krebshilfe.de/

- http://www.krebs-praedisposition.de/

- http://clincancerres.aacrjournals.org/

pediatricseries

- https://www.mh-hannover.de/

humangenetik.html

entlastet werden. Ein Ergebnis der genetischen Diagnostik kann sein, dass pathogene Varianten de novo entstanden sind. Auch in diesem Fall können pathogene Varianten an Nachkommen der betroffenen Person weitergegeben werden. Auf die Möglichkeit eines Keimbahnmosaiks bei einem Elternteil ist in der genetischen Beratung hinzuweisen. Für alle Verwandten, die die pathogene Variante tragen, ist ein intensiviertes Früherkennungsprogramm zu empfehlen. Dies gilt z. B., wenn ein Kind mit einem Medulloblastom eine pathogene Variante im BRCA2-Gen von der gesunden Mutter geerbt hat, die damit ein Risiko von bis zu 80\% trägt, im Laufe des Lebens an Krebs zu erkranken.

\section{Fazit für die Praxis}

- Etwa 5-10\% der Krebserkrankungen von Kindern entstehen auf Basis einer genetischen Krebsprädisposition.

- In der Praxis unterstützt ein Fragebogen bei der Entscheidung, ob der Verdacht auf eine genetische Krebsprädisposition gestellt werden sollte. Neben der Anamnese werden hier auch pathologische Befunde und somatische Genveränderungen berücksichtigt.

- Patienten mit Verdacht auf oder Diagnose einer Krebsprädisposition sollten interdisziplinär betreut werden. Die Diagnose kann von Relevanz für die aktuelle Behandlung, die zukünftige Versorgung und die Familienplanung sein.

- Gesunden Angehörigen können nach einer Beratung prädiktive genetische Untersuchungen angeboten werden.
- In Abhängigkeit von der Krebsprädisposition sowie von Alter und Geschlecht der Betroffenen sind speziell intensivierte Früherkennungsuntersuchungen und gegebenenfalls prophylaktische Operationen indiziert. Ziel ist es weitere Krebserkrankungen zu verhindern beziehungsweise möglichst früh zu diagnostizieren.

\section{Korrespondenzadresse}

\section{Prof. Dr. B. Schlegelberger}

Institut für Humangenetik, Medizinische Hochschule Hannover

Carl-Neuberg-Straße 1, 30625 Hannover, Deutschland

Schlegelberger.Brigitte@mh-hannover.de

\section{Einhaltung ethischer Richtlinien}

Interessenkonflikt. T. Ripperger und B. Schlegelberger geben an, dass kein Interessenkonflikt besteht.

Dieser Beitrag beinhaltet keine von den Autoren durchgeführten Studien an Menschen oder Tieren.

The supplement containing this article is not sponsored by industry.

Open Access. Dieser Artikel wird unter der Creative Commons Namensnennung 4.0 International Lizenz (http://creativecommons.org/licenses/by/4.0/deed. de) veröffentlicht, welche die Nutzung, Vervielfältigung, Bearbeitung, Verbreitung und Wiedergabe in jeglichem Medium und Format erlaubt, sofern Sie den/die ursprünglichen Autor(en) und die Quelle ordnungsgemäß nennen, einen Linkzur Creative Commons Lizenz beifügen und angeben, ob Änderungen vorgenommen wurden.

\section{Literatur}

1. Agaimy A, Foulkes WD (2018) Hereditary SWI/SNF complex deficiency syndromes. Semin Diagn Pathol 35:193-198

2. Churchman ML, Qian M, Te Kronnie G, Zhang R, Yang W, Zhang $\mathrm{H}$, Lana T, Tedrick P, Baskin R, Verbist K, Peters JL, Devidas M, Larsen E, Moore IM, Gu Z, Qu C, Yoshihara H, Porter SN, Pruett-Miller SM, Wu G, Raetz E, Martin PL, Bowman WP, Winick N, Mardis E, Fulton R, Stanulla M, Evans WE, Relling MV, Pui CH, Hunger SP, Loh ML, Handgretinger R, Nichols KE, Yang JJ, Mullighan CG (2018) Germline Genetic IKZF1 Variation and Predisposition to Childhood Acute Lymphoblastic Leukemia. Cancer Cell 33:937-948e8

3. Grobner SN, Worst BC, Weischenfeldt J, Buchhalter I, Kleinheinz K, Rudneva VA, Johann PD, Balasubramanian GP, Segura-Wang M, Brabetz S, Bender S, Hutter B, Sturm D, PfaffE, Hubschmann D, Zipprich G, Heinold M, Eils J, Lawerenz C, Erkek S, Lambo S, Waszak S, Blattmann C, Borkhardt A, Kuhlen M Eggert A, Fulda S, Gessler M, Wegert J, Kappler R, Baumhoer D, Burdach S, Kirschner-Schwabe
R, Kontny U, Kulozik AE, Lohmann D, Hettmer S, Eckert C, Bielack S, Nathrath M, Niemeyer C, Richter GH, Schulte J, Siebert R, Westermann F, Molenaar JJ, Vassal G, Witt H, Project IP-S, Project IM-S, Burkhardt B, Kratz CP, Witt O, Van Tilburg CM, Kramm CM, Fleischhack G, Dirksen U, Rutkowski S, Fruhwald M, Von Hoff K, Wolf S, Klingebiel T, Koscielniak E, Landgraf P, Koster J, Resnick AC, Zhang J, Liu Y, Zhou X, Waanders AJ, Zwijnenburg DA, Raman P, Brors B, Weber UD, Northcott PA, Pajtler KW, Kool M, Piro RM, Korbel JO, Schlesner M, Eils R, Jones DTW, Lichter P, Chavez L, Zapatka M, Pfister SM (2018) The landscape of genomic alterations across childhood cancers. Nature 555:321-327

4. Jarvinen HJ, Aarnio M, Mustonen $\mathrm{H}$, Aktan-Collan $\mathrm{K}$, Aaltonen LA, Peltomaki P, De La Chapelle A, Mecklin JP (2000) Controlled 15-year trial on screening for colorectal cancer in families with hereditary nonpolyposis colorectal cancer. Gastroenterology 118:829-834

5. Jongmans MC, Loeffen JL, Waanders E, Hoogerbrugge PM, Ligtenberg MJ, Kuiper RP, Hoogerbrugge N (2016) Recognition of genetic predisposition in pediatric cancer patients: an easy-to-use selection tool. Eur J Med Genet 59:116-125

6. Mosse YP, Laudenslager $M$, Longo $L$, Cole KA, Wood A, Attiyeh EF, Laquaglia MJ, Sennett $R_{\text {, }}$ Lynch JE, Perri P, Laureys G, Speleman F, Kim C, Hou C, Hakonarson H, Torkamani A, Schork NJ, Brodeur GM, Tonini GP, Rappaport E, Devoto M, Maris JM (2008) Identification of ALK as a major familial neuroblastoma predisposition gene. Nature 455:930-935

7. Oldridge DA, Wood AC, Weichert-Leahey $N$, Crimmins I, Sussman R, Winter C, Mcdaniel LD, Diamond M, Hart LS, Zhu S, Durbin AD, Abraham BJ, Anders L, Tian L, Zhang S, Wei JS, Khan J, Bramlett K, Rahman N, Capasso M, lolascon A, Gerhard DS, Guidry Auvil JM, Young RA, Hakonarson H, Diskin SJ, Look AT, Maris JM (2015) Genetic predisposition to neuroblastoma mediated by a LMO1 superenhancer polymorphism. Nature 528:418-421

8. Ripperger T, Beger C, Rahner N, Sykora KW, Bockmeyer CL, Lehmann U, Kreipe HH, Schlegelberger B (2010) Constitutional mismatch repair deficiency and childhood leukemia/lymphoma-report on a novel biallelic MSH6 mutation. Haematologica 95:841-844

9. Ripperger T, Bielack SS, Borkhardt A, Brecht IB, Burkhardt B, Calaminus G, Debatin KM, Deubzer $H$, Dirksen U, Eckert C, Eggert A, Erlacher M, Fleischhack G, Fruhwald MC, Gnekow A, Goehring G, Graf N, Hanenberg H, Hauer J, Hero B, Hettmer S, Von Hoff K, Horstmann M, Hoyer J, Illig T, Kaatsch P, Kappler R, Kerl K, Klingebiel T, Kontny U, Kordes U, Korholz D, Koscielniak E, Kramm CM, Kuhlen M, Kulozik AE, Lamottke B, Leuschner I, Lohmann DR, Meinhardt A, Metzler M, Meyer LH, Moser O, Nathrath M, Niemeyer CM, Nustede R, Pajtler KW, Paret C, Rasche M, Reinhardt D, Riess O, Russo A, Rutkowski S, Schlegelberger B, Schneider D, Schneppenheim R, Schrappe M, Schroeder C, Von Schweinitz D, Simon T, SparberSauer M, Spix C, Stanulla M, Steinemann D, Strahm B, Temming P, Thomay K, Von Bueren AO, Vorwerk P, Witt O, Wlodarski M, Wossmann W, Zenker M, Zimmermann S, Pfister SM, Kratz CP (2017) Childhood cancer predisposition syndromes-A concise review and recommendations by the Cancer Predisposition Working Group of the Society for Pediatric Oncology and Hematology. Am J Med Genet A 173:1017-1037

10. Ripperger T, Hofmann W, Koch JC, Shirneshan $K$, Haase D, Wulf G, Issing PR, Karnebogen M, 
Schmidt G, Auber B, Schlegelberger B, Illig T, Zirn B, Steinemann D (2018) MDS1 and EVI1 complex locus (MECOM): a novel candidate gene for hereditary hematological malignancies. Haematologica 103:e55-e58

11. Ripperger T, Steinemann D, Gohring G, Finke J, Niemeyer CM, Strahm B, Schlegelberger B (2009) A novel pedigree with heterozygous germline RUNX1 mutation causing familial MDS-related AML: can these families serve as a multistep model for leukemic transformation? Leukemia 23:1364-1366

12. Ripperger $T$, Tauscher $M$, Haase $D$, Griesinger F, Schlegelberger B, Steinemann D (2011) Managing individuals with propensity to myeloid malignancies due to germline RUNX1 deficiency. Haematologica 96:1892-1894

13. Ripperger T, Tawana K, Kratz C, Schlegelberger B, Fitzgibbon J, Steinemann D (2016) Clinical utility gene card for: familial platelet disorder with associated myeloid malignancies. Eur J Hum Genet. https://doi.org/10.1038/ejhg.2015.278

14. Schlegelberger B, Kreipe H, Lehmann U, Steinemann D, Ripperger T, Gohring G, Thomay K, Rump A, Di Donato N, Suttorp M (2015) A child with LiFraumeni syndrome: Modes to inactivate the second allele of TP53 in three different malignancies. Pediatr Blood Cancer 62:1481-1484

15. Shah S, Schrader KA, Waanders E, Timms AE, Vijai J, Miething C, Wechsler J, Yang J, Hayes J, Klein RJ, Zhang J, Wei L, Wu G, Rusch M, Nagahawatte P, Ma J, Chen SC, Song G, Cheng J, Meyers P, Bhojwani D, Jhanwar S, Maslak P, Fleisher M, Littman J, Offit L, Rau-Murthy R, Fleischut MH, Corines M, Murali R, GaoX, ManschreckC, Kitzing T, Murty VV, Raimondi S, Kuiper RP, Simons A, Schiffman JD, Onel K, Plon SE, Wheeler D, Ritter D, Ziegler DS, Tucker K, Sutton $R$, Chenevix-Trench G, Li J, Huntsman DG, Hansford S, Senz J, Walsh T, Lee M, Hahn CN, Roberts K, King MC, Lo SM, Levine RL, Viale A, Socci ND, Nathanson KL, Scott HS, Daly M, Lipkin SM, Lowe SW, Downing $J R$, Altshuler D, Sandlund JT, Horwitz MS, Mullighan CG, Offit K (2013) A recurrent germline PAX5 mutation confers susceptibility to pre-B cell acute lymphoblastic leukemia. Nat Genet 45:1226-1231

16. Stanulla M, Dagdan E, Zaliova M, Moricke A, Palmi C, Cazzaniga G, Eckert C, Te Kronnie G, Bourquin JP, Bornhauser B, Koehler R, Bartram CR, Ludwig WD, Bleckmann K, Groeneveld-KrentzS, ScheweD, Junk SV, Hinze L, Klein N, Kratz CP, Biondi A, Borkhardt A, Kulozik A, Muckenthaler MU, Basso G, Valsecchi MG, Izraeli S, Petersen BS, Franke A, Dorge P, Steinemann D, Haas OA, Panzer-Grumayer R, Cave $\mathrm{H}$, Houlston RS, Cario G, Schrappe M, Zimmermann M, Consortium T, International BFMSG (2018) IKZF1(plus) defines a new minimal residual disease-dependent very-poor prognostic profile in pediatric B-cell precursor acute lymphoblastic leukemia. JClin Oncol 36:1240-1249

17. Tolbert VP, Coggins GE, Maris JM (2017) Genetic susceptibility to neuroblastoma. Curr Opin Genet Dev 42:81-90

18. Vasen HF, Ghorbanoghli Z, Bourdeaut $F$, Cabaret O, Caron O, Duval A, Entz-Werle N, Goldberg $\mathrm{Y}$, llencikova D, Kratz CP, Lavoine N, Loeffen J, Menko FH, Muleris M, Sebille G, Colas C, Burkhardt B, Brugieres L, Wimmer K, Cmmr-D EU-CCF (2014) Guidelines for surveillance of individuals with constitutional mismatch repair-deficiency proposed by the European Consortium "Care for CMMR-D" (C4CMMR-D). J Med Genet 51:283-293

19. Waszak SM, Northcott PA, Buchhalter I, Robinson GW, Sutter C, Groebner S, Grund KB, Brugieres L, Jones DTW, Pajtler KW, Morrissy AS, Kool M,
Sturm D, Chavez L, Ernst A, Brabetz S, Hain $M$, Zichner T, Segura-Wang M, Weischenfeldt J, Rausch T, Mardin BR, Zhou X, Baciu C, Lawerenz C, Chan JA, Varlet P, Guerrini-Rousseau L, Fults DW, Grajkowska W, Hauser P, Jabado N, Ra YS, Zitterbart K, Shringarpure SS, De La Vega FM, Bustamante $\mathrm{CD}, \mathrm{Ng} \mathrm{HK}$, Perry A, Macdonald TJ, Hernaiz Driever P, Bendel AE, Bowers DC, Mccowage G, Chintagumpala MM, Cohn R, Hassall T, Fleischhack G, Eggen T, Wesenberg F, Feychting M, Lannering B, Schuz J, Johansen C, Andersen TV, Roosli M, Kuehni CE, Grotzer M, Kjaerheim K, Monoranu CM, Archer TC, Duke E, Pomeroy SL, Shelagh R, Frank S, Sumerauer D, Scheurlen W, Ryzhova MV, Milde T, Kratz CP, Samuel D, Zhang J, Solomon DA, Marra M, Eils R, Bartram CR, Von Hoff K, Rutkowski S, Ramaswamy V, Gilbertson RJ, Korshunov A, Taylor MD, Lichter P, Malkin D, Gajjar A, Korbel JO, Pfister SM (2018) Spectrum and prevalence of genetic predisposition in medulloblastoma: a retrospective genetic study and prospective validation in a clinical trial cohort. Lancet Oncol 19:785-798

20. Wlodarski MW, Hirabayashi S, Pastor V, Stary J, Hasle H, Masetti R, Dworzak M, Schmugge $M$, Van Den Heuvel-Eibrink M, Ussowicz M, De Moerloose B, Catala A, Smith OP, Sedlacek P, Lankester AC, Zecca M, Bordon V, Matthes-Martin $S$, Abrahamsson J, Kuhl JS, Sykora KW, Albert MH, Przychodzien B, Maciejewski JP, Schwarz S, Gohring G, Schlegelberger B, Cseh A, Noellke P, Yoshimi A, Locatelli F, Baumann I, Strahm B, Niemeyer CM, Ewog MDS (2016) Prevalence, clinical characteristics, and prognosis of GATA2related myelodysplastic syndromes in children and adolescents. Blood 127:1387-1397 (quiz 1518) 